Original Paper http://ajol.info/index.php/ijbcs http://indexmedicus.afro.who.int

\title{
Analyse des paramètres du spermogramme en relation avec le fructose, le citrate et l'alpha glucosidase neutre du sperme chez les hommes consultant pour infertilité à Libreville
}

\author{
Alvine Sybille BATOU ${ }^{1}$, Félix OVONO ABESSOLO ${ }^{1 *}$, Immaculée MBA ${ }^{1}$ et \\ Armel MINTSA ${ }^{2}$ \\ ${ }^{1}$ Laboratoire de Biochimie, Faculté de Médecine et des Sciences de la Santé, BP. 4009 Libreville - Gabon \\ ${ }^{2}$ Laboratoire National de Santé Publique, Libreville - Gabon \\ *Auteur correspondant ; BP 4009 Libreville, Gabon ; E-mail: ovonab@yahoo.fr; \\ Tel : 00.241.07.07.70.49
}

\section{RESUME}

L'infertilité est un problème de santé publique dans les pays en voie de développement et son exploration onéreuse chez l'homme repose surtout sur la cytologie du sperme, sans l'associer à la biochimie du sperme. Ainsi une relation entre les anomalies du spermogramme et l'alpha-glucosidase, le fructose et le citrate du plasma séminal, a été rechercher, afin de justifier ou non la rareté de la biochimie du sperme. Une étude transversale et descriptive, s'est déroulée d'octobre 2017 à mars 2018 inclus, au laboratoire National de Santé Publique de Libreville. Tous les patients admis consécutivement pendant la période d'étude, pour effectuer un spermogramme pour bilan d'infertilité, ont été inclus. Le sperme, obtenu au laboratoire par masturbation, a servi aux analyses macroscopiques et microscopiques. L'alpha-glucosidase neutre, le fructose et le citrate ont été dosés dans le plasma séminal. L'analyse univariée a permis de choisir les paramètres à inclure dans l'analyse factorielle des correspondances entre les paramètres cytologiques et biochimiques. Sur le plan du spermogramme, les anomalies retrouvées étaient nombreuses et associées chez un même malade, eu égard à l'indice d'anomalies multiples. L'anomalie cytologique prédominante était l'oligoasthénotératonécrozoospermie $(40,0 \%)$, suivie de l'oligoasthénotératospermie $(7,3 \%)$. Sur le plan biochimique, l'apha-glucosidase était basse chez $21,8 \%$ des patients, alors que le citrate était abaissé chez $20,0 \%$ des sujets. Seuls $1,8 \%$ des sujets présentaient une baisse du taux de fructose. Par ailleurs, les anomalies biochimiques n'étaient pas associées aux anomalies du spermogramme. Le profil cytologique du spermogramme de cette population est dominé par les associations d'anomalies. Cependant, comme ces paramètres ne sont pas associés aux paramètres biochimiques du plasma séminal, il est indispensable de prescrire ces deux types d'analyses pour une meilleure prise en charge des sujets consultant pour infertilité. (C) 2018 International Formulae Group. All rights reserved.

Mots clés: spermogramme, infertilité, fructose, citrate, alpha-glucosidase

\begin{abstract}
Infertility is a public health problem in developing countries and its expensive exploration in male relies mainly on the cytology of sperm, without association with seminal biochemistry. Thus, a relationship between spermogram abnormalities and alpha-glucosidase, fructose and seminal plasma citrate has been investigated, in order to justify or not the rarity of sperm biochemistry. A cross-sectional and descriptive study
\end{abstract}


was conducted from October 2017 to March 2018, at the Libreville National Public Health Laboratory. All patients admitted consecutively during the study period, to perform a spermogram for infertility assessment, were included. Sperm, obtained in the laboratory by masturbation, was used for macroscopic and microscopic analyzes. Neutral alpha-glucosidase, fructose and citrate were obtained in seminal plasma. Univariate analysis made it possible to choose the parameters to be included in the factorial analysis between cytological and biochemical parameters. In terms of spermogram, abnormalities found were numerous and associated in the same patient, given the multiple anomalies index. The predominant cytologic abnormality was oligoasthenoterato-necrozoospermia $(40.0 \%)$, followed by oligoasthenoteratospermia $(7.3 \%)$. For biochemical tests, apha-glucosidase was low in $21.8 \%$ of patients, while citrate was lowered in $20.0 \%$ of subjects. Only $1.8 \%$ of subjects had a decrease in fructose levels. In addition, biochemical abnormalities were not associated with abnormalities of the spermogram. Associations of anomalies dominate the cytological profile of the spermogram of this population. However, as these parameters are not associated with the biochemical parameters of the seminal plasma, it is essential to prescribe these two types of analysis for a better support of the subjects consulting for infertility.

(C) 2018 International Formulae Group. All rights reserved.

Keywords: Spermogram, infertility, fructose, citrate, alpha-glucosidase.

\section{INTRODUCTION}

L'infertilité est définie comme l'incapacité pour un couple d'aboutir à une grossesse clinique, après 12 mois de rapports sexuels réguliers et non protégés (ZegersHoschild et al., 2017). Il s'agit d'un problème de santé publique mondiale, avec une prévalence estimée à $15 \%$ des couples dans le monde (Sharlip et al., 2002). L'Afrique n'est pas en reste, car il a été estimé que l'infertilité affecte $15-30 \%$ des couples mariés dans les pays africains (Umeora et al., 2007). Le Gabon fait partie de la ceinture d'infertilité avec des taux particulièrement élevés, allant de 14 à 20\% des couples (Sembuya, 2010). Comme dans d'autres régions, l'infertilité est un problème social, qui entraine une stigmatisation de la femme (Tabong et Adongo, 2013). Dans peu de cas, l'homme accepte de se remettre en cause (Parrott, 2014). En effet, la composante masculine dans l'infertilité du couple a été estimée en Afrique subsaharienne à 20-40\% (Agarwal, 2015). Pour ces hommes qui acceptent de consulter pour infertilité, en dehors du bilan hormonal, il est préconisé la réalisation d'une analyse de sperme, sous forme de spermogramme et spermocytogramme (Cooper et al., 2010; Esteves et al., 2012). Par ailleurs, une analyse biochimique du sperme peut également contribuer dans l'exploration de l'infertilité masculine (Feng et al., 2015). Cependant, dans notre contexte, la biochimie du sperme n'est pas encore largement utilisée par les praticiens. Cette étude dont le but est de décrire la répartition des anomalies du spermogramme et des paramètres biochimiques du plasma séminal chez les sujets consultant pour infertilité à Libreville et de rechercher une relation entre ces deux types de paramètres a été entreprise.

\section{MATERIEL ET METHODES}

Ce travail a été réalisé en respectant les règles édictées dans les recommandations de Helsinki (Association Médicale Mondiale, 2013). Ces règles portaient notamment sur la confidentialité des données, la liberté pour chaque sujet d'accepter ou non de participer à l'étude et la possibilité de s'y retirer à tout moment de son déroulement. Il s'agit d'une étude descriptive et ponctuelle qui s'est effectuée au service de Bactériologie du Laboratoire National et au laboratoire de Biochimie de l'Université des Sciences de la Santé d'Owendo, d'octobre 2017 à mars 2018. Les patients qui se sont présentés avec une prescription de spermogramme avec pour motif le bilan d'infertilité, ont été consécutivement inclus. Ces patients 
bénéficiaient ensuite et gratuitement, de la réalisation de la biochimie du sperme. Les patients dont le motif était autre que le bilan d'infertilité et ceux qui refusaient de participer à l'étude n'étaient pas inclus. Le spermogramme se faisait à l'issue d'un rendez-vous qui était fixé 4 jours avant l'examen, afin de respecter le délai d'abstinence des rapports sexuels, du tabac et de l'alcool.

Les paramètres sociodémographiques et cliniques de chaque patient ont été recueillis sur une fiche. Le jour de l'examen, les antécédents médicaux (infections chlamydienne, ourlienne, gonococcique; diabète, hypertension artérielle) et chirurgicaux (chirurgie du tractus urogénitale) ainsi que la consommation énolotabagique ont été précisés. Le prélèvement se faisait au laboratoire dans une pièce prévue à cet effet, par masturbation, après un lavage soigneux des mains et de la verge. La totalité du sperme était recueilli dans un réceptacle stérile qui était par la suite identifié.

Les analyses macroscopiques et microscopiques du sperme ont été faites selon les recommandations de l'OMS (WHO, 2010). Les caractères macroscopiques comme le volume du sperme, son aspect (épais, fluide ou léger), sa coloration (blanchâtre ou jaunâtre), son opacité (faible, moyenne ou forte) et sa viscosité (normale ou anormale) ont été notés. Sur le plan microscopique, les paramètres recherchés étaient la concentration spermatique, la vitalité (\%), la mobilité à une heure (totale, progressive rapide, progressive lente et non progressive) et à quatre heures. Les éléments cellulaires ont été également recherchés : les hématies, les cellules rondes et les leucocytes. Après le décompte des spermatozoïdes dont la morphologie était normale, les spermatozoïdes ont été examinés, en commençant par l'acrosome puis la tête, la pièce intermédiaire et le flagelle. L'index d'anomalies multiples a été obtenu, puis décompte de la mobilité totale du sperme a été calculé selon l'équation suivante:

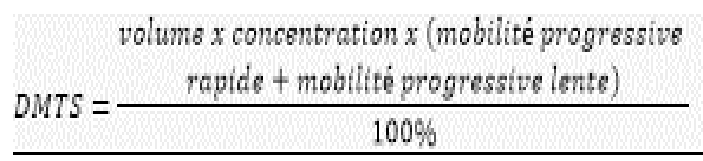

Les analyses de fructose, de citrate et de l'alpha-glucosidase neutre ont été effectuées sur le plasma séminale, à l'aide des FertiPro®, selon les recommandations du fabriquant.

\section{Analyse des données}

Les variables qualitatives ont été exprimées en pourcentage et les quantitatives en moyenne \pm écart-type. Le test non paramétrique de Mann Whitney-Wilcoxson a permis de comparer les variables quantitatives entre groupes; les corrélations univariées ont permis de choisir les variables quantitatives corrélées aux paramètres biochimiques. $\mathrm{Ce}$ sont ces dernières qui ont été utilisées pour l'analyse factorielle des correspondances, qui a été effectuée sans orthogonalisation. Les deux premiers axes factoriels de cette analyse ont été retenus. Les différences étaient considérées comme significatives pour une probabilité $\mathrm{p}<0,05$.

\section{RESULTATS}

Durant la période d'étude 144 patients avaient pris rendez-vous et $114 \quad(79,2 \%)$ s'étaient présentés pour le prélèvement. Parmi ces patients, quatre n'avaient pas pu éjaculer au laboratoire, ce qui a ramené l'échantillon à 110. L'âge moyen de ces patients était de $39,5 \pm 7,9$ ans. Ces sujets avaient déjà en moyenne 1,7 12,6 enfants avec des extrêmes allant de 0 à 13 enfants (Tableau 1). Il a été noté que $49,1 \%$ de ces patients consommaient de l'alcool et $12,2 \%$ se déclaraient fumeurs. Dans cette population, 4 (3,6\%) présentaient des antécédents de chirurgie dont l'un une cure de varicocèle bilatérale et l'autre une 
cure herniaire unilatérale. Il y avait 9,1\% patients étaient diabétiques. Ici, $69,1 \%$ avaient des antécédents infectieux soit $27,3 \%$ de chlamydiose, $21,8 \%$ de gonococcie et $20 \%$ d'oreillons. Par ailleurs, $70,9 \%$ de la population avaient au moins un facteur de risque d'infertilité contre $29,1 \%$ qui n'avaient aucun antécédent.

Les résultats de l'analyse macroscopique du sperme ont été consignés dans le Tableau 2. Dans cette étude, le volume moyen du sperme était de 3,9 $\pm 3,9 \mathrm{ml}$ avec des extrêmes allant de 0,8 à $30 \mathrm{ml}$. Douze patients $(10,9 \%)$ présentaient une anomalie de volume dont $4(3,6 \%)$ hypospermies et $8(7,3 \%)$ hyperspermies. Le sperme de $61,8 \%$ patients était fluide et le $\mathrm{pH}$ ne dépassait pas les limites de la normale. Le sperme était de coloration jaunâtre dans $18,2 \%$ des cas et dans $36,4 \%$ des cas il était de viscosité anormale.

Les résultats de l'analyse microscopique du sperme ont été consignés dans le Tableau 3. La concentration spermatique moyenne était de $23,9.10^{6} \pm 35,2.10^{6}$ spermatozoïdes $/ \mathrm{ml}$. Aucune polyzoospermie n'avait été observée alors que 4 patients $(3,6 \%)$ avaient présenté une azoospermie. La vitalité moyenne était de $40,1 \pm 17,8 \%$ avec des extrêmes allant de 0 à $76 \%$. Cette vitalité était mauvaise dans $81,8 \%$ des cas. Les mobilités totales moyennes à une et à quatre heures étaient respectivement de $36,2 \pm 17,2 \%$ et $26,3 \pm 18,5 \%$ avec des extrêmes allant de 0 à $70 \%$. La recherche d'hématies dans le sperme était positive dans $54,5 \%$ des cas, la concentration moyenne en leucocytes était de $7,0 \pm 7,4 \cdot 10^{3} / \mathrm{ml}$. La concentration moyenne en cellules rondes était de $2,5 \pm 2,6.10^{3} / \mathrm{ml}$. Dans ce travail $10,9 \%$ des spermes comportaient entre 0 et 500 cellules rondes/ml, la même proportion présentait entre 5001 et 16000 cellules rondes/ml. Les agglutinations étaient absentes dans 7,3\% des spermes, rares dans $27,3 \%$ et nombreuses dans $32,7 \%$ des spermes analysés. Quelques cellules épithéliales ont été retrouvées dans
$47,3 \%$ des spermes, alors qu'elles étaient absentes dans $14,5 \%$ des cas et nombreuses dans $16,4 \%$ (Figure 1). Du point de vu morphologique, seuls $12,7 \%$ de spermes comprenaient une proportion de spermatozoïdes normaux compris entre 51 et $70 \%$. Dans $16,4 \%$ des spermes le nombre de spermatozoïdes normaux variait de 0 à $10 \%$ (Figure 2).

Les proportions des anomalies de l'acrosome et des dimensions de la tête des spermatozoïdes ont été résumées dans le Tableau 4. Dans ce travail, 27,3\% des spermes comportaient entre 9 et $16 \%$ de spermatozoïdes ayant des anomalies de l'acrosome. Par contre, 34,5\% des spermes avaient entre 3 et $5 \%$ de spermatozoïdes présentant des anomalies de l'acrosome. Par ailleurs, 27,3\% des spermes présentaient entre 14 et $25 \%$ des spermatozoïdes macrocéphales. Alors que $14,5 \%$ des spermes analysés montraient entre 12 et $20 \%$ de spermatozoïdes avec une microcéphalie. Il a été retrouvé dans $3,6 \%$ des spermes une proportion de 16 à $20 \%$ des spermatozoïdes à tête effilée. Dans $20 \%$ des spermes contenaient 10 à $16 \%$ de spermatozoïdes avaient une tête irrégulière. Par contre, 76,4\% des spermes montraient 0$2 \%$ de spermatozoïdes à tête multiple.

Les anomalies observées sur la pièce intermédiaire et la queue des spermatozoïdes ont été consignées dans le Tableau 5. Dans $20 \%$ des spermes analysés un taux de 7 à $15 \%$ des spermatozoïdes présentait des anomalies de la base de la pièce intermédiaire. Par ailleurs, dans $12,7 \%$ des spermes, 10 à $16 \%$ des spermatozoïdes avaient conservés des restes cytoplasmiques. L'anomalie de la queue la plus rare était représentée par la queue multiple avec une fréquence de $0,65 \pm 1,60 \%$. Ainsi $81,8 \%$ des spermes présentaient $0-1 \%$ des spermatozoïdes à tête multiple. $43,6 \%$ des spermes ne montrait que $0-2 \%$ des spermatozoïdes sans queue. Les résultats de l'étude de l'index d'anomalies multiples (IAM) ont été résumés dans la Figure 3. En 
moyenne, cet index était de 1,44 $\pm 0,42$ avec les extrêmes allant de 0 à 2,3. La plage d'IAM allant de 1,51 à 1,75 était la plus fréquente $(30,9 \%)$. Par contre celle allant de 0 à 1 n'atteignait que $7,3 \%$ de la population étudiée.

Les principaux diagnostics retenus après analyse macroscopique et microscopique ont été résumés dans le Tableau 6. Ainsi $92,7 \%$ des spermes présentaient au moins une anomalie. Seuls $7,3 \%$ des patients présentaient une normospermie et la même proportion présentait une hyperspermie et une oligoasthéno-thératospermie. L'oligo-asthénotérato-nécrosospermie était rencontré chez $40 \%$ des sujets suivie de l'asthénotératonécrospermie $(10,9 \%)$ et de l'oligoasthénotératospermie $\quad(7,3 \%) . \quad$ Par contre, $3,6 \%$ des patients avaient une azoospermie isolée, la même proportion présentait une hypospermie.

Sur le plan biochimique, l'activité moyenne de l'alpha-glucosidase était de $34,1 \pm 17,1 \mathrm{mUI} /$ éjaculat avec des extrêmes allant de 6,2 à 101,9\%. La quantité moyenne de fructose était de 29,4 $\pm 24,4 \mathrm{mg} /$ éjaculat, alors que la quantité de citrate moyenne de $18,2 \pm 16,9 \mathrm{mg} /$ éjaculat. Au cours de cette étude, $21,8 \%$ des patients avaient un taux d'alpha-glucosidase inférieur à la normale. Dans 20\% des cas, les patients présentaient un taux de citrate inférieur à la normale et dans $1,8 \%$ des cas on retrouvait un taux de fructose inférieur à la normale. Par contre, 8 spermes $(7,3 \%)$ avaient simultanément une baisse de citrate et d'alphaglucosidase.

Les résultats de l'analyse factorielle associant les paramètres cytologiques et biochimiques sont représentés sous forme de carte factorielle à la Figure 4. Cette analyse avait mis en évidence une opposition sur le premier axe, entre l'âge et le nombre de spermatozoïdes, leur mobilité totale à une heure et quatre heures, leur vitalité et le décompte de la mobilité totale du sperme. Par contre, sur le second axe, le citrate, le volume et l'alphaglucosidase se retrouvaient regroupés.

Tableau 1 : Paramètres épidémiologiques des patients.

\begin{tabular}{ll}
\hline Paramètres socio-démographiques & Résultats \\
\hline Population totale N $(\%)$ & $110(100)$ \\
Age $($ ans $)(\mathrm{m} \pm \mathrm{ET})$ & $39,5 \pm 7,9$ \\
Nombre d'enfants $(\mathrm{m} \pm \mathrm{ET})$ & $1,7 \pm 2,6$ \\
Professions N $(\%)$ & \\
$\quad$ Enseignants & $16(14,5)$ \\
Autres fonctionnaires & $20(18,2)$ \\
Commerçants & $18(16,4)$ \\
Privées & $30(27,3)$ \\
Sans profession & $26(23,6)$ \\
Antécédents N $(\%)$ & \\
Alcool & $54(49,1)$ \\
Tabac & $14(12,7)$ \\
Diabète & $10(9,1)$ \\
Chlamydiose & $30(27,3)$ \\
Gonococcie & $24(21,8)$ \\
Oreillons & $22(20,0)$ \\
\hline
\end{tabular}


Tableau 2 : Résultats de l'analyse macroscopique du sperme de l'ensemble de l'échantillon.

\begin{tabular}{ll}
\hline Paramètres & Résultats \\
\hline Volume $(\mathrm{ml}) \mathrm{m} \pm \mathrm{ET}$ & $3,9 \pm 3,9$ \\
$\mathrm{pH}(\mathrm{m} \pm \mathrm{ET})$ & $7,7 \pm 0,4$ \\
Aspect N $(\%)$ & \\
Epais & $34(30,9)$ \\
Fluide & $68(61,8)$ \\
Léger & $8(7,3)$ \\
Couleur N $(\%)$ & \\
Blanchâtre & $90(81,8)$ \\
Jaunâtre & $20(18,2)$ \\
Opacité N $(\%)$ & \\
Faible & $26(23,6)$ \\
Moyenne & $62(56,4)$ \\
Forte & $22(20,0)$ \\
Normale & $70(63,6)$ \\
Anormale & $40(36,4)$ \\
\hline
\end{tabular}

Tableau 3 : Résultats de l'analyse microscopique du sperme de tous les patients.

\begin{tabular}{lr}
\hline Paramètres & \multicolumn{1}{c}{ Valeurs } \\
\hline Concentration spermatique $(/ \mathrm{ml})(\mathrm{m} \pm \mathrm{ET})$ & $28,0.10^{6} \pm 42,7.10^{6}$ \\
Oligospermie N $(\%)$ & 58,8 \\
Polyzoospermie $(\%)$ & $40,1 \pm 17,8$ \\
Vitalité $(\% \mathrm{~m} \pm \mathrm{ET})$ & \\
Mobilité à une heure $(\% \mathrm{~m} \pm \mathrm{ET})$ & $36,2 \pm 17,2$ \\
$\quad$ Mobilité totale & $13,7 \pm 12,2$ \\
Mobilité progressive rapide & $12,2 \pm 6,5$ \\
Mobilité progressive lente & $10,4 \pm 5,4$ \\
Mobilité non progressive & \\
$\quad$ Mobilité totale & $26,3 \pm 18,5$ \\
Mobilité progressive rapide & $8,4 \pm 10,0$ \\
Mobilité progressive lente & $9,1 \pm 5,8$ \\
Mobilité non progressive & $8,6 \pm 5,3$ \\
Mobilité à quatre heures $\%$ m \pm ET) & $836,3 \pm 1381,3$ \\
Concentration d'Hématies $(\mathrm{m} \pm \mathrm{ET}) / \mathrm{ml}$ & $2,5.10^{3} \pm 2,6.10^{3}$ \\
Concentration des Cellules rondes $(\mathrm{m} \pm \mathrm{ET}) / \mathrm{ml}$ & $7,0.10^{3} \pm 7,4.10^{3}$ \\
Concentration des Leucocytes $(\mathrm{m} \pm \mathrm{ET}) / \mathrm{ml}$ & $1,4 \pm 0,4$ \\
Index d'anomalies multiples $(\mathrm{m} \pm \mathrm{ET})$ & $29,3 \pm 16,8$ \\
Morphologie normale $(\% \mathrm{~m} \pm \mathrm{ET})$ & \\
\hline
\end{tabular}




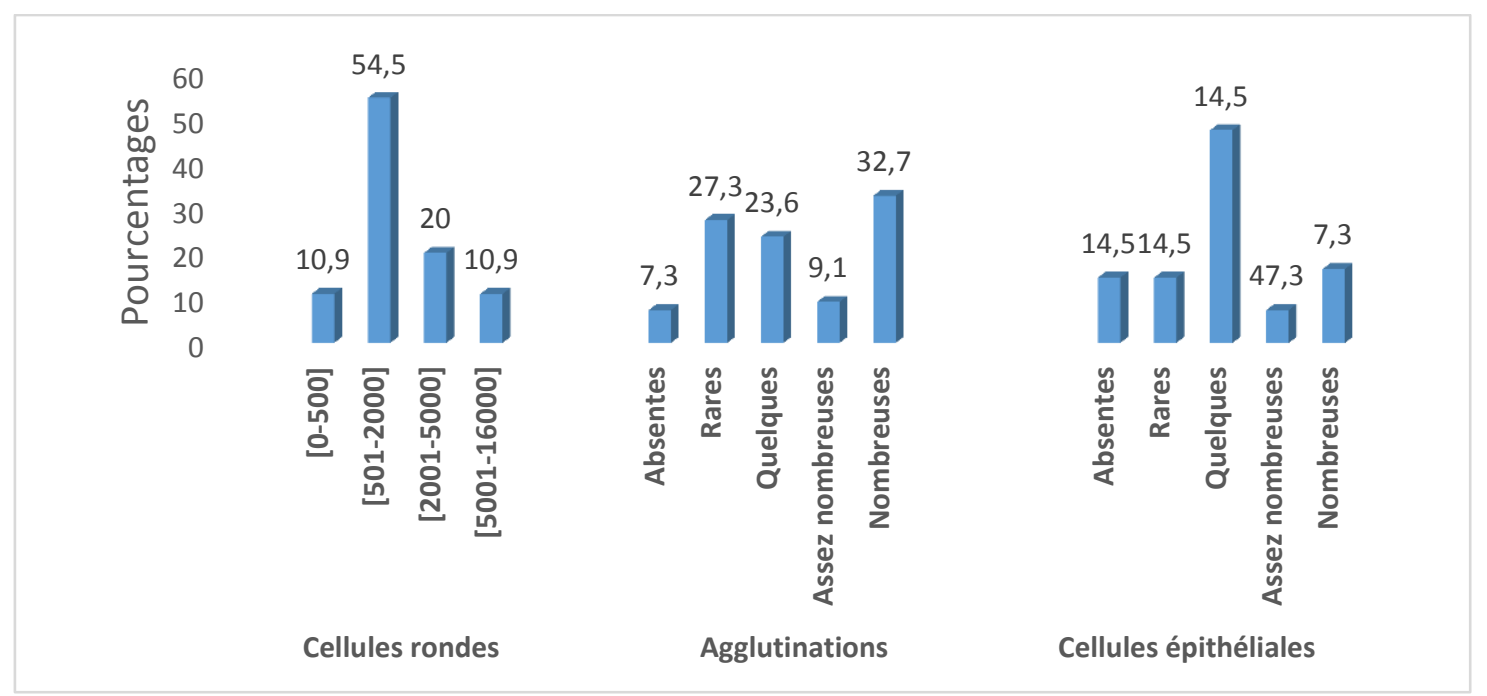

Figure 1: Répartition des proportions de cellules rondes, agglutinations et cellules épithéliales dans les spermes.

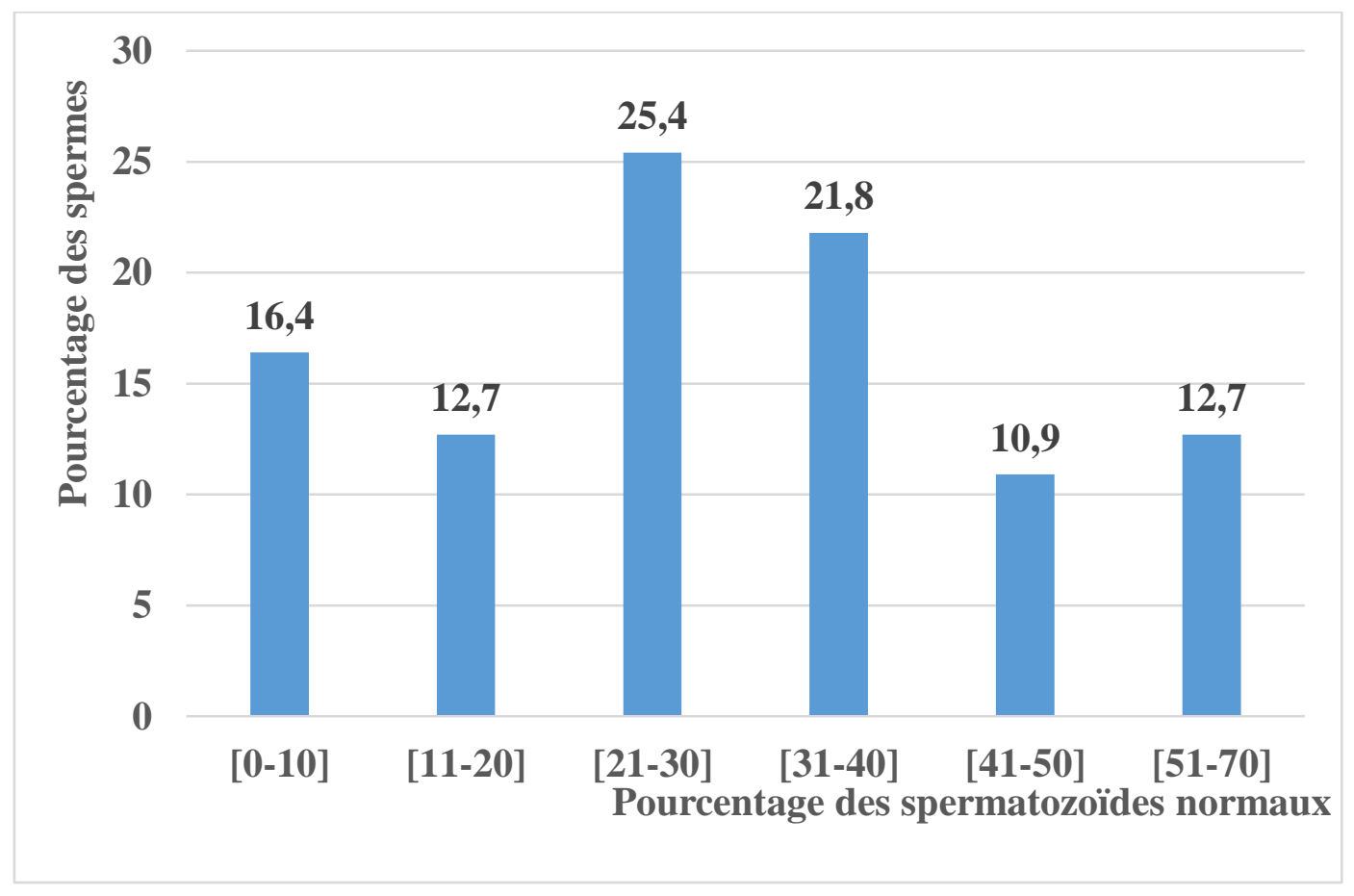

Figure 2 : Répartition des pourcentages de spermes ayant des spermatozoïdes de morphologie normale. 
Tableau 4 : résumé des anomalies de l'acrosome et de la tête des spermatozoïdes.

\begin{tabular}{|c|c|}
\hline Paramètres & Valeurs N (\%) \\
\hline \multicolumn{2}{|c|}{$\begin{array}{l}\text { Proportion des spermatozoïdes ayant une anomalie de l'acrosome } \\
(\%)\end{array}$} \\
\hline$[0-2]$ & $20(18,2)$ \\
\hline$[3-5]$ & $38(34,5)$ \\
\hline$[6-8]$ & $22(20,0)$ \\
\hline$[9-16]$ & $30(27,3)$ \\
\hline \multicolumn{2}{|c|}{ Proportions des spermatozoïdes ayant une tête effilée (\%) } \\
\hline$[0-5]$ & $52(49,1)$ \\
\hline$[6-10]$ & $36(32,7)$ \\
\hline$[11-15]$ & $16(14,6)$ \\
\hline$[16-20]$ & $4(3,6)$ \\
\hline \multicolumn{2}{|c|}{ Proportion des spermatozoïdes ayant une tête irrégulière (\%) } \\
\hline$[0-3]$ & $22(20,0)$ \\
\hline$[4-6]$ & $38(34,5)$ \\
\hline$[7-9]$ & $28(25,5)$ \\
\hline$[10-16]$ & $22(20,0)$ \\
\hline \multicolumn{2}{|c|}{ Proportion des spermatozoïdes ayant une tête multiple (\%) } \\
\hline$[0-2]$ & $84(76,4)$ \\
\hline$[3-6]$ & $24(21,8)$ \\
\hline$[7-8]$ & $2(1,8)$ \\
\hline \multicolumn{2}{|c|}{ Proportion des spermatozoïdes ayant une macrocéphalie (\%) } \\
\hline$[0-6]$ & $42(38,2)$ \\
\hline$[7-9]$ & $10(9,1)$ \\
\hline$[10-13]$ & $28(25,4)$ \\
\hline$[14-25]$ & $30(27,3)$ \\
\hline \multicolumn{2}{|c|}{ Proportion des spermatozoïdes ayant une microcéphalie (\%) } \\
\hline$[0-5]$ & $30(27,3)$ \\
\hline$[6-8]$ & $30(27,3)$ \\
\hline$[9-11]$ & $34(30,9)$ \\
\hline$[12-20]$ & $16(14,5)$ \\
\hline
\end{tabular}

Tableau 5 : résultat de l'analyse de la pièce intermédiaire et de la queue des spermatozoïdes.

\section{Paramètres}

Valeurs N (\%)

Proportion des spermatozoïdes ayant une anomalie de la base (\%)

$$
\begin{aligned}
& {[0-3]} \\
& {[4-6]} \\
& {[7-15]}
\end{aligned}
$$

Proportions des spermatozoïdes ayant des restes cytoplasmiques $(\%)$

$$
\begin{aligned}
& {[0-2]} \\
& {[3-5]}
\end{aligned}
$$




$$
\begin{aligned}
& {[6-9]} \\
& {[10-15]}
\end{aligned}
$$

Proportions des spermatozoïdes ayant une queue écourtée (\%)

$$
\begin{aligned}
& {[0-4]} \\
& {[5-7]} \\
& {[8-12]} \\
& {[13-38]}
\end{aligned}
$$$$
6(5,4)
$$

Proportions des spermatozoïdes ayant une queue enroulée (\%)

$$
\begin{aligned}
& {[0-2]} \\
& {[3-5]} \\
& {[6-8]} \\
& {[9-12]}
\end{aligned}
$$

Proportions des spermatozoïdes ayant une queue multiple (\%)

$$
\begin{aligned}
& {[0-1]} \\
& {[2-3]} \\
& {[4-10]}
\end{aligned}
$$

$4(3,6)$

Proportions des spermatozoïdes n'ayant pas de queue (\%)

$$
\begin{aligned}
& {[0-2]} \\
& {[3-5]} \\
& {[6-8]} \\
& {[9-35]}
\end{aligned}
$$

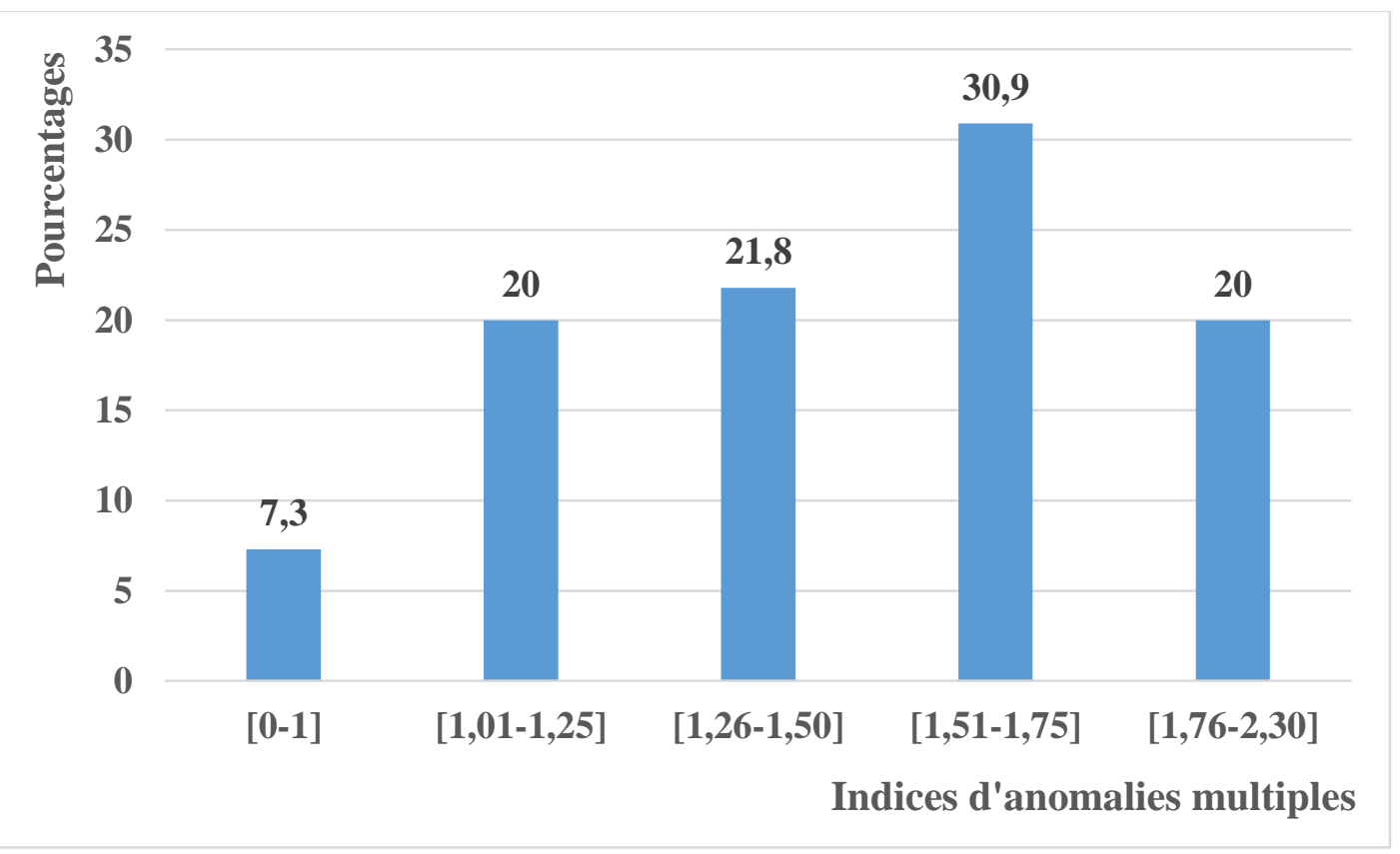

Figure 3 : Distribution de l'Index d'Anomalies Multiples. 
Tableau 6: répartition des diagnostics retenus lors de l'analyse du spermogramme et du spermocytogramme.

\begin{tabular}{lr}
\hline Anomalies & Valeur (\%) \\
\hline Oligoasthénotératonécrozoospermie & $44(40,0)$ \\
Asthénotératonécrozoospermie & $11(10,0)$ \\
Oligoasthénotératospermie & $8(7,3)$ \\
Normospermie & $8(7,3)$ \\
Oligoasthénonécrozoospermie & $6(5,5)$ \\
Asthénospermie & $6(5,5)$ \\
Asthénonécrozoospermie & $4(3,6)$ \\
Azoospermie & $4(3,6)$ \\
Hypospermie & $4(3,6)$ \\
Oligoasthénospermie & $4(3,6)$ \\
Oligotératospermie & $4(3,6)$ \\
Asthénotératospermie & $2(1,8)$ \\
Nécrotératozoospermie & $2(1,8)$ \\
Oligonécrozoospermie & $2(1,8)$ \\
Tératozoospermie & $2(1,8)$ \\
\hline
\end{tabular}

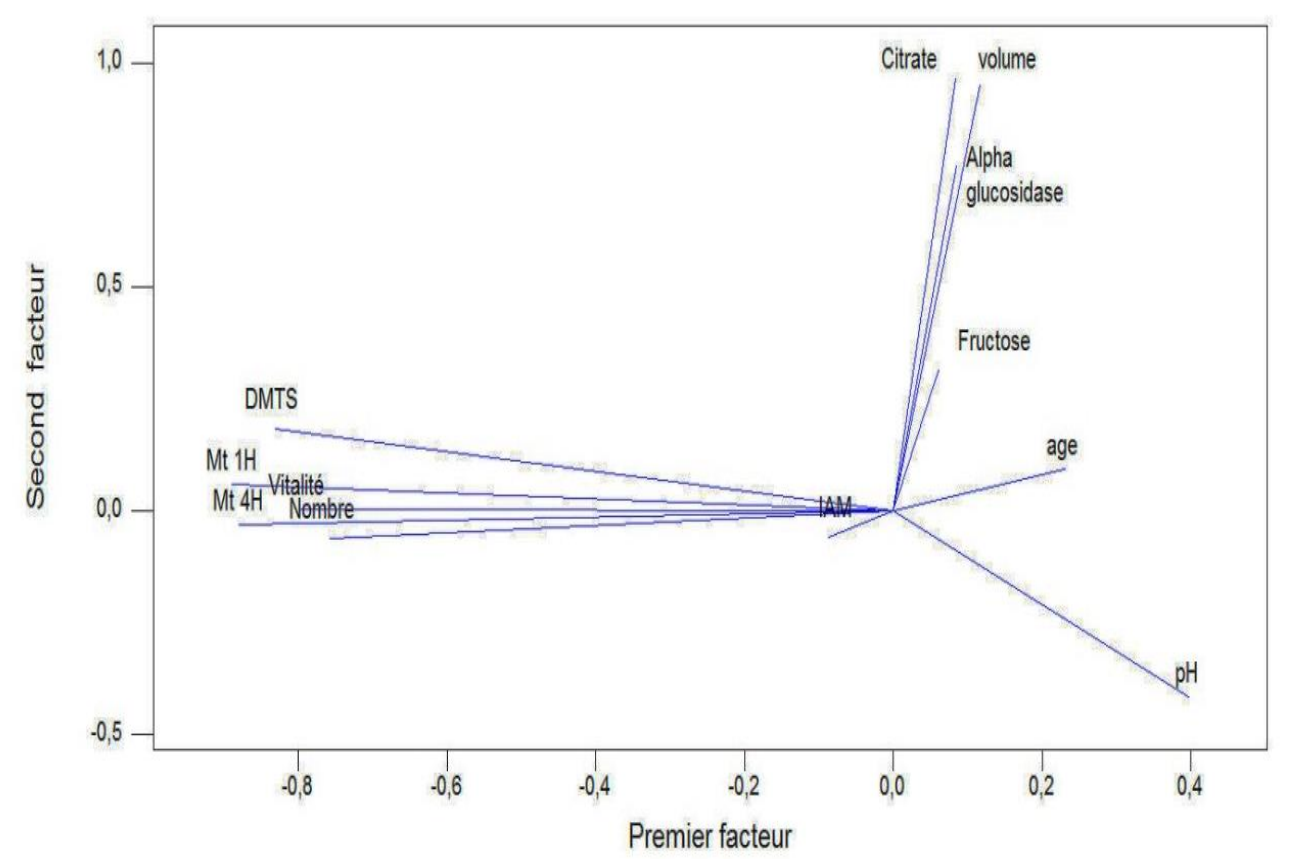

Figure 4: analyse factorielle des paramètres cytologiques et biochimiques (\% de variance expliquée 64\%). 


\section{DISCUSSION}

L'infertilité masculine est un sujet encore mal exploré dans notre région où en effet, le spermogramme et le bilan hormonal sont systématiquement prescrits sans tenir compte de l'analyse des composantes du plasma séminal. L'objectif de ce travail était de décrire la répartition des anomalies du spermogramme et des paramètres biochimiques du plasma séminal chez les sujets consultant pour infertilité, et de rechercher une relation entre ces deux types de paramètres.

Au cours de cette étude, 144 patients avaient pris rendez-vous pour la réalisation du spermogramme et seuls $114(79,2 \%)$ ont honoré ce rendez-vous. Cela serait dû à la confusion par certains hommes entre virilité et fécondité. Ils pensent que le fait d'éjaculer est synonyme de fécondité. En effet, en Afrique, peu d'hommes acceptent d'être à l'origine de l'infertilité dans un couple (Parrott, 2014). Cela explique leur réticence à consulter et préfèrent voir ce problème comme un tabou ou alors un problème mystique (Moyo, 2013). Or la contribution masculine à l'infertilité du couple, peut aller jusqu'à $50 \%$ ou même plus (Vander Borght and Wyns, 2018). Ceci est d'autant plus difficile à admettre que cette infertilité peut être due à des déficiences testiculaires ou post-testiculaires et par conséquent toucher à la virilité de l'homme. Quatre (4) patients n'avaient pas pu éjaculer au laboratoire et certains avaient du mal à le faire. Ces derniers étaient venus seuls alors qu'ils vivaient en couple. Or, la présence de la partenaire pourrait rendre facile l'éjaculation. Cette difficulté d'éjaculer serait liée à la pression psychologique c'est-à-dire, un certain niveau de stress à canaliser et à l'effort de l'acte de masturbation (Moyo, 2013 ; Vander Borght and Wyns, 2018). Cette relation a été mise en évidence par Price et al. (2005) en démontrant que les hommes vivant en couple avaient plus de difficultés à obtenir du sperme par masturbation que ceux qui n'avaient pas de partenaires.

L'âge moyen de la population d'étude était de 39,5 57,9 ans. Cette tranche d'âge correspond à celle où les questions de fertilité deviennent préoccupantes. Cet âge moyen est plus élevé que celui trouvé dans l'étude de Martin Odoom et al. (2015) qui était de 24-36 ans, par contre proche de ceux trouvé par d'OH et al. (2015) et par Samake à Bamako (2007). De même, El Bardisi et al. (2018) avaient trouvé cet âge de 35 à 36 ans, confirmant donc que les hommes consultent généralement pour infertilité à la trentaine. Dans ce travail le nombre d'enfants des patients variait entre 0 et 13 . Cela prouve qu'une consultation pour infertilité est envisageable même en ayant des enfants; dans ce cas il serait question d'une stérilité secondaire. Cependant, comme l'a démontré Matetakufa (1998), devant une infertilité du couple, une femme peut aussi contracter une grossesse avec un autre homme que son partenaire, avec ou sans l'accord de ce dernier dans le but de mettre fin à la dérision que représente l'infertilité du couple. D'ailleurs, il n'est pas exceptionnel qu'un père de famille apprenne à l'hôpital qu'il est totalement infertile. Il a été mis en évidence quatre (4) patients $(3,6 \%)$ avec des antécédents de chirurgie du tractus uro-génital: l'un une varicocèlectomie bilatérale et l'autre une cure herniaire unilatérale. Or, l'influence de la varicocèle sur l'infertilité masculine n'est plus à démontrer. $\mathrm{La}$ varicocèle altère la spermatogénèse par atteinte des cellules de Leydig. Elle diminue également la concentration de testostérone par atteinte des cellules de Sertoli (Nervoux et al., 2009). La varicocèle entraine également une élévation des dérivés actifs de l'oxygène et du monoxyde d'azote, dans le plasma séminal (Köksal et al., 2000 ; Sakamoto et al., 2008). $\mathrm{OH}$ et al. (2015) en recherchant les causes d'infertilité avaient trouvé $20,9 \%$ des patients présentant une varicocèle. Bayasgalan $\mathrm{D}$ et al. (2004) ont également trouvé des chiffres avoisinant ceux de $\mathrm{OH}$ et al. (2015) c'est-àdire 18,4\%. Par ailleurs, $27,3 \%$ de la population avaient des antécédents de chlamydiose. Or il a été démontré que cette dernière, comme de nombreuses autres affections bactériennes sexuellement transmissibles, est impliquée dans l'infertilité masculine (Bayasgalan et al., 2004 ; Al- 
Moushaly, 2013; Dehghan et al., 2016). En effet, ces infections seraient responsables de l'inflammation des voies urogénitales et de la production de radicaux libres oxygénés. Comme l'avaient démontré Azenabor et al. (2015), l'inflammation entraine un remaniement architectural des cellules du tractus atteint, qui à son tour, serait responsable d'une perturbation des fonctions des glandes accessoires, du transport des spermatozoïdes ou de la spermatogénèse. En effet, de nombreux auteurs (Tawadrous et al., 2013 ; Methorst et al., 2014 ; Roychoudhury et al., 2016 ; Agarwal et al., 2016) ont prouvé que le spermatozoïde était une cellule particulièrement sensible au stress oxydant. Ceci a pour conséquences une altération des paramètres du spermogramme, dégradation de l'ADN et parfois la formation de varicocèle.

La population d'étude était composée de $49,1 \%$ de patients consommant de l'alcool. Ce dernier est une source exogène de dérivés réactifs de l'oxygène par l'augmentation de leur production. Il interfère aussi avec les mécanismes de défense antioxydante de l'organisme. Il est donc considéré comme facteur de risque de stérilité masculine ( $\mathrm{La}$ Vignera et al., 2013). Pour cette raison, le sevrage est l'un des éléments clé dans la prise en charge de cette infertilité masculine, (De Jong et al., 2014 ; Guthauser et al., 2014), tout comme le sevrage tabagique. En effet, Lotti et al. (2015) ont démontré que le tabagisme entrainait une diminution du volume $\mathrm{du}$ sperme. Paradoxalement, nous n'avons retrouvé que $12,2 \%$ de fumeurs. Mais dans tous les cas, la consommation d'alcool et de tabac sont des éléments délétères pour la production et la maturation des spermatozoïdes. Le simple sevrage de quelques jours que nous avons institué avant le prélèvement n'est probablement pas suffisant pour que les effets de l'alcool et du tabac ne soient pas perçus.

Nous avons trouvé 92,3\% de spermes présentant au moins une anomalie au spermogramme. Ce résultat est proche de celui de Sakande et al. (2012) $(84,1 \%)$. Sur le plan macroscopique, $30,9 \%$ des spermes étaient épais, associé au 36,4\% dont la viscosité était anormale. Or de la viscosité du plasma séminale dépend l'efficacité des mouvements du flagelle, en vue d'assurer la fécondité de l'œuf. Ces chiffres sont d'ailleurs à rapprocher de la mobilité totale à une heure $(36,2 \pm 17,2)$, et sont attendus à des valeurs aussi élevées dans une population de sujets infertiles, avec une vitalité moyenne de $40,1 \pm 17,8$. Le volume moyen du sperme était de $3,9 \pm 3,9 \mathrm{ml}$ cette valeur est proche de celle trouvée par Saïdi et al. (2008) $(4,08 \pm 10,23$ $\mathrm{ml})$. Il a été retrouvé six patients $(10,9 \%)$ avec des anomalies du volume du sperme. Cette valeur est proche de Sakande et al. (2012) qui avait retrouvé $19 \%$ d'anomalie de volume. Cela fait part de la relative rareté des anomalies de volume chez nos patients consultant pour infertilité, car Sakande avait trouvé 19\% (2012). Aucun sperme ne présentait une anomalie de $\mathrm{pH}$. Une acidité ferait penser à une insuffisance ou atteinte prostatique. Cette dernière serait responsable d'une anomalie de mobilité comme l'ont démontré d'autres études notamment celles de Zhou et al. (2015) et Jimenez et al. (2010). Un $\mathrm{pH}$ basique ferait penser à une atteinte des vésicules séminales.

La concentration spermatique moyenne était de $28,0 \pm 42,7.10^{6} / \mathrm{ml}$ pour un taux d'oligospermie de $58,8 \%$. La diminution du nombre de spermatozoïdes a trois principales causes. Les causes pré-testiculaires qui sont liées aux niveaux hormonaux sécrétés par l'hypophyse (Gordetsky et al., 2011). Les causes testiculaires qui sont liées à des lésions telles qu'une varicocèle (El Bardisi, 2018) et les causes post-testiculaires liées à une obstruction ou absence congénital des conduits déférents Ho et al (2015). L'inflammation de l'urètre peut également empêcher l'expulsion des spermatozoïdes. La numération des spermatozoïdes dans ce travail retrouve une moyenne plus élevée que celle trouvée par Saïdi et al. (2008) $(20,44 \pm 3,12)$. Owolabi et al. (2016) au Nigeria, ont trouvé un taux d'oligospermie de $26,6 \%$ dans leur population d'étude, soit pratiquement la moitié des nôtres, ce qui prouve la fréquence élevée de cette anomalie en cas d'infertilité. Bien que le nombre de spermatozoïdes ne soit 
pas le critère prédictif de grossesse spontanée le plus important, son rôle est néanmoins indéniable. Il parait donc logique que le nombre de spermatozoïdes soit faible dans une population infertile, comme celle qui fait l'objet de notre étude. Dans ce sens, c'est le décompte de la mobilité totale du sperme qui est le critère prédictif le plus important pour une grossesse spontanée (Hamilton et al., 2015). Ceci se retrouve avec les relations entre ce paramètre et les autres éléments de la cytologie du sperme, comme la mobilité, la vitalité et même la concentration spermatique.

Dans ce travail, $80 \%$ des spermes avec une vitalité inferieure à la normale pour une

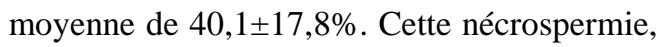
serait liée à la fréquence des infections urogénitales. En effet, Zinzendorf et al. (2008) ont mis en évidence une prévalence d'Ureaplasma urealyticum chez 39\% des spermes des sujets infertiles à Abidjan en Côte d'Ivoire. Cette vitalité est abaissée par rapport à l'étude de Saïdi et al. (2008) qui avait trouvé $72,49 \pm 1,70 \%$ en Israël. La morphologie des spermatozoïdes n'est pas à omettre. En effet, la morphologie normale moyenne était de $29,3 \% \pm 16,8$, avec un index d'anomalies multiples allant de 0 à 2,3. Ces anomalies seraient dues à la fréquence des infectieuses du tractus urogénital dans la population d'étude. Elles peuvent également être d'origine hormonale ou toxique. Ce résultat est opposé à celui trouvé par Bayasgalan et al. (2004) en Mongolie $(1,2 \%)$ et de ceux de Sakande et al. (2012) à Ouagadougou $(9,1 \%)$. Il a été retrouvé 4 patients $(3,6 \%)$ avec une azoospermie. Cette dernière peut être d'origine sécrétoire puisqu'aucun de ces patients ne présentait de trouble au niveau des dosages biochimiques, ou alors excrétoire. Cette azoospermie peut être d'origine pré-testiculaire (secondaire à une absence de production de FSH), testiculaire (liée à une lésion testiculaire) ou post-testiculaire (excrétoire) liée à une obstruction des canaux déférents. Ces résultats sont différents de ceux d'autres auteurs (Bayasgalan et al., 2004; Sakande et al., 2012 ; Ho et al., 2015 ; Owalabi et al., 2016). Mais dans tous les cas, l'azoospermie est rare.
Seuls trois patients $(7,3 \%)$ présentaient une normospermie. Cela montre l'extrême rareté de spermes normaux dans notre population d'étude, contrairement à Owolabi al. (2016) qui ont trouvé $68,2 \%$ de normospermie au Nigeria. Par contre, la fréquence des anomalies est à mettre en parallèle avec la prédominance des diagnostics associés, dont le principal est l'oligoasthénotératonécrozoospermie (OATN) à $40 \%$. Ces anomalies sont non seulement nombreuses mais aussi et surtout associées entre elles. En outre, un sperme présente simultanément une anomalie de nombre de spermatozoïdes, de la mobilité et de la vitalité. Cette intrication serait due au fait que dans notre population d'étude, $70,9 \%$ des patients présentaient au moins un facteur de risque d'infertilité sans oublier qu'il existe aussi des facteurs génétiques et immunologiques. Ce résultat n'est pas similaire à celui de l'étude de Owolabi et al. (2016) au Nigeria qui ont trouvé un taux de $2,1 \%$ d'OATN et Sakande et al. (2012) à Ouagadougou qui a aussi trouvé 13,6\% d'OATN. Une certitude apparait ici, la variabilité des paramètres des spermes en fonction des régions comme l'ont souligné El bardisi et al. (2018). Cette différence serait due à l'exposition à des produits toxiques, comme l'ont souligné Dalvie et al. (2004), des différences ethniques (Khandwala et al., 2017), sans oublier les habitudes et modes de vie tel que le tabagisme (Sharma et al., 2016).

En analyse multivariée, il apparait une opposition entre l'âge et les paramètres de la fertilité (mobilité, vitalité, nombre de spermatozoïdes et décompte de la mobilité totale). En d'autres termes l'âge apparait comme un facteur limitant de la fertilité. Johnson et al. (2015) ont également démontré cette relation. Même si l'intervalle d'âge de notre population n'est pas très important $(39,5 \pm 7,9$ ans $)$. Ceci suggère que les consultations pour infertilité masculine devraient se faire plus précocement, afin de bénéficier encore de quelques paramètres de qualité. Dans cette étude, nous n'avons pas tenu compte d'autres facteurs de variabilité des paramètres du spermogramme, comme l'indice de masse corporelle. Alors qu'il est 
connu qu'il s'agit d'un facteur impliqué dans l'infertilité (Sermondale et al., 2013 ; Luque et al., 2015).

L'absence de l'indice de masse corporelle et la variabilité de l'analyse du spermogramme elle-même explique la variabilité de $64 \%$ des deux premiers axes de l'analyse factorielle, même si cette analyse a été effectuée selon les recommandations de l'OMS (WHO, 2010). Comme marqueur épididymaire, l'alpha-glucosidase neutre était abaissée dans le sperme de $21,8 \%$ des sujets de cette étude, contre $20 \%$ pour le citrate et seulement $1,8 \%$ pour le fructose, or le fructose est la principale source d'énergie du spermatozoïde. Ce résultat suggère que les anomalies de mobilité sont dues à d'autres phénomènes et non à un déficit en fructose, du moins pour ce travail. Par ailleurs, les paramètres cytologiques et biochimiques du sperme ne sont pas associés dans ce travail. Ce résultat a été trouvé par Said et al. (2008). L'explication de cette absence de relation serait due au fait que les anomalies cytologiques sont multiples, associées les unes aux autres chez un même sujet, comme en témoigne l'index des anomalies multiples. Ceci justifie la prescription des analyses cytologiques et biochimiques dans l'étude de l'infertilité chez l'homme.

\section{Conclusion}

Malgré la taille de notre échantillon, les anomalies cytologiques mises en évidence dans cette population de sujets consultant pour infertilité masculine, sont très variées et associées les unes autres, d'où la prédominance de l'oligoasthénotératonécrozoospermie. Cependant, la relation entre ces anomalies et les valeurs des paramètres biochimiques du plasma séminal n'a pas été mise en évidence. Ceci justifierait la prescription simultanée du spermogramme et de ces paramètres biochimiques, afin d'identifier les étiologies de l'infertilité de cette population, et d'y apporter les thérapeutiques les plus appropriées. De plus, une étude de plus grande envergure et prenant en compte d'autres paramètres du plasma séminal comme le zinc, la fragmentation de
l'ADN et la recherche d'anticorps antispermatozoïdes serait nécessaire pour préciser ce résultat.

\section{CONFLIT D'INTERETS}

Les auteurs ne déclarent aucun conflit d'intérêt.

\section{CONTRIBUTIONS DES AUTEURS}

BAS a effectué les prélèvements et réalisé les analyses cytologiques et biochimiques; OAF a rédigé le protocole, et validé la version finale du manuscrit; MI a réalisé les prélèvements, les analyses cytologiques et biochimiques et rédigé le manuscrit; MA a corrigé et validé le manuscrit.

\section{REFERENCES}

Agarwal A, Mulgund A, Hamada A, Chyatte MR. 2015. A unique view on male infertility around the globe. Reproductive Biology and Endocrinology, 13: 37.

Agarwal A, Roychoudhury S, Bjugtad KB, Cho CL. 2016. Oxidation-reduction potential of semen: what is its role in the treatment of male infertility? Therapeutic Advances in Urology, 8(5): 302-318. DOI : $10.1177 / 1756287216652779$.

Al-Moushaly A. 2013. Recent acquisitions in the medical treatment of infertility caused by the Chlamydiae trachomatis. Journal of Medecine and Life, 6(2):168170.

Association Médicale Mondiale. 2013. Principes éthiques applicables à la recherche médicale impliquant des êtres humains ; Helsinki. Disponible sur http://www.wma.net/fr/. Consulté le 24/05/2018.

Azenabor A, Ekun AO, Akinloye O. 2015. Impact of Inflammation on Male Reproductive Tract. Journal of Reproduction and Infertility, 16(3): 123129.

Bayasgalan G, Naranbat D, Radnaabazar J, Lhagvasuren T, Rowe PJ. 2004. Male infertility: risk factors in Mongolian 
men. Asian Journal of Andrology, 6(4): 305-311.

Cooper TG, Noonan E, von Eckardstein S, Auger J, Baker HW, Behre HM, Haugen TB, Kruger T, Wang C, Mbizvo MT, Vogelsong KM. 2010. World Health Organization reference values for human semen characteristics. Human Reproduction Update, 16: 231245. DOI: 10.1093/humupd/dmp048.

Dalvie MA, Myers JE, Thompson ML, Robins TG, Dyer S, Riebow J, Molekwa J, Jeebhay M, Millar R, Kruger P. 2004. The long-term effects of DDT exposure on semen, fertility and sexual function of malaria vector-control workers in Limpopo province South Africa. Environmental Research, 96: 1-8. DOI :10.1016/j.envres.2003.09.002.

De Jong AM, Menkveld R, Len JW, Nienhuis SE, Rhemrev JP. 2014. Effect of alcohol intake and cigarette smoking on sperm parameters and pregnancy. Andrologia, 43(2): 112-117. DOI:10.1111/and.12054.

Dehghan Marvast L, Aflatoonian A, Talebi AR, Ghasem-Zadeh J, Pacey AA. 2016. Semen inflammatory markers and Chlamydiae trachomatis infection in male partners of infertile couples. Andrologia, 48(7): 729-736. DOI: 10.1111/and.12501.

El bardisi H, Maj zoub A, Al Said S, Al Rumaihi K, El Ansari W, Allatar A, Arafa M. 2018. Geographical differences in semen characteristics of 13892 infertile men. Arab Journal of Urology, 16: 3-9. DOI: org/10.1016/j.aju.2017.11.018.

Esteves SC, Zini A, Aziz N, Alvarez JG, Sabanegh ES Jr, Agarwal A. 2012. Critical appraisal of world organization reference values for human semen characteristics and effect on diagnosis health and treatment of subfertile men. Urology, 79(1): 16-22. DOI: 101016/j.urology.2011.08.003.

Feng RX, Lu JC, Lu NG. 2015. A pilot comparative study of 26 biochemical markers in seminal plasma and serum in infertile men. BioMed Research
International, 2015: 805326. DOI: $10.1155 / 2015 / 805328$.

Gordetsky J, Wijngaarden EV, O’Brien J. 2011. Redefining abnormal folliclestimulating hormone in the male infertility population. BJU International, 110(4) : $\quad 568-572 . \quad$ DOI: 10.1111/j.1464.410X.2011.10783x.

Guthauser B, Boitrelle F, Plat A, Thiercelin N, Vialard F. 2014. Chronic excessive alcohol consumption and male fertility: a case report on reversible azoospermia and a literature review. Alcohol Alcohol, 49(1): 42-44. DOI: 10.1093/alcalc/agt133.

Hamilton JAM, Cissen M, Brandes M, Smeenk JMJ, deBruin JP, Kremer JAM, Nelen WLDM, Hamilton CJCM. 2015. Total motile sperm count a better indicator for the severity of male factor infertility than the WHO sperm classification system. Human Reproduction, 30(5): 1110-1121. DOI: 10.1093/humrep/dev058.

Ho KL, Tsu JH, Tamp PC, Yiu MK. 2015. Disease spectrum and treatment patterns in a local male infertility clinic. Hong Kong Medical Journal, 21(1): 5-9.

Jimenez T, Sanchez G, Wertheimer E, Blanco G. 2010. Activity of the Na,K-ATPase alpha4isoform is important for membrane potential, intracellular $\mathrm{Ca}^{2+}$ and $\mathrm{pH}$ to maintain motility in rat spermatozoa. Reproduction, 139(5): 835845. DOI: 10.1530/REP-09-0495.

Johnson SH, Dunleavy J, Gemmell NJ, Nabagawa S. 2015. Consistent agedependent declines in human semen quality: a systematic review and metaanalysis. Ageing Research Reviews, 19: 22-33. DOI: 10.1016/j.arr.2014.10.007

Khandwala YS, Zhang CA, Li S, Belir B, Quo D, Eisenberg ML. 2017. Racial variation in semen quality at fertility evaluation. Urology, 106: 96-102. DOI: 10.1016/j.urology.2017.03.064.

Köksal IT, Tefekli A, Usta M, Erol H, Abbasoglu S, Kadioglu A. 2000. The role of reactive oxygen species in testicular dysfunction associated with varicocele. BJU Int, 86: 549-552. 
La Vignera S, Condorelli RA, Balercia G, Vicari E, Calogero AE. 2013. Does alcohol have any effect on male reproductive function? A review of literature. Asian Journal of Andrology, 15(2): 221-225. DOI: 10.1038/aja.2012.118.

Lotti F, Corona G, Vitale P, Maseroli E, Rossi M, Fino MG, Maggi M. 2015. Current smoking is associated with lower seminal vesicles and ejaculate volume, despite higher testosterone levels, in male subjects of infertile couples. Human Reproduction, 30(3): 590-602. DOI: 10.1093/humrep/deu347.

Luque EM, Tissera A, Gaggino MP, Molina RI, Mangeand A, Vincenti LM, Beltramore F, Sad Larcher J, Estofan D, Fiol de Cuneo M, Martini AC. 2017. Body mass index and human sperm quality: neither one extreme nor the other. Reproduction, Fertility and Development, 29(4): 731-739. DOI: 10.1071/RD15351.

Martin-Odoom A, Brown CA, Adjei DN. 2015. Level of male infertility in the Ghanaian city of Tema. Journal of Obstetrical Gynaecology, 35(8): 825828.

DOI: 10.3109/01443615.2015.1009876.

Matetakufa SN. 1998. Infertility: our own gift. New Internationalist.

McLachlan RI. 2000. The endocrine control of spermatogenesis. Clinical Endocrinology and Metabolism, 14(3): 345-362.

DOI : 10.1053/beem.2000.0084.

Methorst C, Huyghe E, membres du Comité d'Andrologie et de Médecine Sexuelle de l'Association Française d'Urologie, Sous-Comité Fertilité masculine du CAMS. 2014. Stress oxydant et infertilité masculine: physiopathologie et intérêt thérapeutique des antioxydants. Progrès en Urologie, 24: 4-10.

Moyo S. 2013. Indigenous knowledge systems and attitudes towards male infertility in Mhondoro-Ngezi, Zimbabwe. Culture Health Sexology, 15(3):667-679. DOI: 10.1080/13691058.2013.779029.
Nervoux P, Robin G, Gonhein T, Boitrelle F, Rigot JM, Marcelli F. 2009 Varicocèle et infertilité: mythe ou réalité? Progrès en Urologie, 19(4): 126-130.

Owalabi AT, Fasubaa OB, Ogunniyi SO. 2016. Semen quality of male partners of infertile couples in Ile-Ife, Nigeria. Nigerian Journal of Clinical Practices, 16(1): 37-40. DOI: $10.4103 / 1119-$ 3077.106729.

Parrott F R. 2014. 'AT the hospital, I learnt the truth: diagnosing male infertility in rural Malawi. Anthropology and Medicine, 21(2): 174-188. DOI: 10.1080/13648470.2014.915618.

Price MA, Cohen MS, Hoffman IF, Chilongozi D, Martinson FE, Tembo T. 2005. Collecting the essence of man: semen collection for HIV transmission studies in sub-saharan Africa. Sexually Transmitted Infections, 81(2): 185-186. DOI: 10.1136/sti.2004.012104.

Roychoudhury S, Sharma R, Sikka S, Agarwal A. 2016. Diagnostic application of total antioxidant capacity in seminal plasma to access oxidatives stress in male factor infertility. Journal of Assisted Reproduction and Genetics; 33(5): 627-635. DOI: 10.1007/s10815016-0677-5.

Said L, Galerand-Denis I, Carreau S, Saad A. 2008. Relationship between semen quality and seminal plasma components: alpha-glucosidase, fructose and citrate in infertile men compared while normospermic population of tunisian men. Andrologia, 41: 150-156. DOI: 10.1111/j.1439-0272.2008.00906.x.

Saïdi R, Rives N, Gruel E, Mazurier S, Mousset-Sméon N, Macé B. 2008. Nouvelle classification du spermocytogramme à fort grossissement. Médecine de la Reproduction, Gynécologie Endocrinologie, 10(5): 315324.

Sakamoto Y, Ishikawa T, Kondo Y, Yamaguchi K and Fujisawa M. 2008. The assessment of oxidative stress in infertile patients with varicocele. $B J U$ International, 101: 1547-1552. DOI: 10.1111/j.1464-410X.2008.07517.x. 
Sakande J, Kabre E, Ekue-Ligan A, Ouédraogo HA, Sawadogo M. 2012. Relation entre les anomalies du spermogramme et les constituants biochimiques du liquide séminal de sujets consultant pour hypofertilité masculine à Ouagadougou. International Journal Biological and Chemical Sciences, 6(3): 1167-1178. DOI: https://dx.doi.org/10.4314/ijbcs.v6i3.22.

Sembuya R. 2010. Mother or nothing: the agony of infertility. Bulletin of the World Health Organization, 88 (12): 881-882. DOI: 10.2471/BLT.10.011210.

Sermondale N, Faure C, Fezen L, Shayeb AG, Bonde JP, Jensen TK, Van Wely M, Cao J, Martini AC, Esbandar M, ChavarroJE, Koloszar S, Twigt JM, Ramlan- Hansen $\mathrm{CH}$, Borges Jr E, Lotti F, SteegersThonnissen RPM, Zoen B, Polotsky AJ, La Vignera S, Esbenazi B, Tremellen K, Magnusdotti EV, Fejes I, Hercberg S, Levy R, Ozernichow S. 2013. BMI in relation to sperm count: an update systematic review and collaborative meta-analysis. Human Reproduction Update, 19(3): 221-231. DOI: 10.1093/humupd/dms050.

Sharlip ID, Jarow JP, Belker AM, Lipshultz LI, Sigman M, Thomas AJ, Schlegel N, Howards SS, Nehra A, Damewood MD, Overstreet JW, Sadovsky R. 2002. Best practice policies for male infertility. Fertility and Sterility; 77:873-82.

Sharma R, Harlev A, Agarwal A, Esteves SC. 2016. Cigarette smoking and semen quality: a now meta-analysis examining the effect of the 2010 World Health Organization laboratory methods for the examination of human semen. European Urology, 70(4): 635-645. DOI: 10.1016/j.eururo.2016.04.010.

Tabong PT, Adongo PB. 2013. Understanding the social meaning of infertility and childbearing: a qualitative study of the perception of childbearing and childlessness in northern Ghana. PLoS
ONE, 8(1): $\quad$ e54429. DOI: 10.1371/journal.pone.0054429.

Tawadrous GA, Aziz AA, Mostafa T. 2013. Seminal soluble fas relationship with oxidative stress in infertile men with varicocele. Urology, 82(4): 820-823. DOI: $10.1016 /$ j.urology.2013.06.018.

Umeora OU, Mbazor JO, Okpere EE. 2007. Tubal factor infertility in Benin city, Nigeria - Sociodemographics of patients and aetiopathogenic factors. Tropical Doctor, 37(2): 92-94. DOI : 10.1177/004947550703700211.

Vander Borght M, Wyns C. 2018. Fertility and infertility: definition and epidemiology. Clinical Biochemistry, pii: S0009-9120(18)30220-0. DOI: 10.1016/j.clinbiochem.2018.03.012

WHO. 2010. Laboratory manual for the examination and processing of human semen; fifth edition.

Zegers-Hochschild F, Adamson GD, Dyer S, Racowsky C, de Mouzon J, Sokol R, Rienzi L, Sunde A, Schmidt L, Cooke ID, Simpson JL, van der Poel S. 2017. The International Glossary on Infertility and Fertility Care, 2017. Fertilility and Sterility, 108(3): 393-406. DOI: 10.1016/j.fertnstert.2017.06.005.

Zhou J, Chen L, Li J, Li H, Hong Z, Xie M, Chen S, Yao B. 2015. Semen pH affects sperm motility and capacitation. PLoS One, 10(7): e0132974. DOI: 10.1371/journal.pone.0132974.

Zinzendorf NY, Kouassi-Agbessi BT, Lathro JS, Don C, Kouadio L, Loukou YG. 2008. Ureaplasma Urealyticum or Mycoplasma Hominis Infections and Semen Quality of Infertile Men in Abidjan. Journal of Reproduction and Contraception, 19(2): 65-72. DOI : doi.org/10.1016/S1001-7844(08)600085. 